\title{
A Proof-of-Principle Experiment of Optical Injection of Electrons in Laser-Driven Plasma Waves
}

\author{
N. Saleh, P. Zhang, S. Chen, Z.-M. Sheng, A. Maksimchuk, V. Yanovsky \\ and D. Umstadter \\ Center for Ultrafast Optical Science, University of Michigan, Ann Arbor, MI 48109, USA
}

\begin{abstract}
We report on a proof-of-principle experiment that demonstrates, for the first time, the feasibility of optically injecting electrons into laser-driven plasma waves, first proposed by [D. Umstadter, J.-K. Kim, and E. Dodd, Phys. Rev. Lett. 76, 2073 (1996)]. Using a Table-Top-Tera Watt laser system (I $\sim 5 \times 10^{18} \mathrm{~W} / \mathrm{cm}^{2}, \lambda=1 \mu \mathrm{m}, \tau=400 \mathrm{fs}$ ), whose output beam is split by 1:4 ratio into a pump and injection beams, respectively, spatial (within $10 \mu \mathrm{m}$ ) and temporal (within $400 \mathrm{fs}$ ) overlap of the two beams were achieved by intersecting them orthogonally in an under-dense $\left(\sim 4 \times 10^{19} \mathrm{~cm}^{-3}\right)$, supersonically produced He plasma jet. The interference of the two beams in the plasma, produces an intensity grid, directed along the bisector of the their propagation directions. This causes the plasma electrons to be trapped and periodically bunched in the intersection region, reaching modulation densities an order of magnitude higher than that of the cold relativistic plasma wave-breaking limit reported so far, and theoretically estimated. As being bunched in the intersection region, the electrons are stochastically heated to temperatures up to a few 100's of $\mathrm{keV}$. This mechanism cooperates with other possible mechanisms to enhance the temperature, current, and emittance of the electron beam produced by the pump pulse alone. A 2-D PIC code simulation corroborates the reported experimental results. Discussed also, is development of a 100-TW-class Ti:Sapphire laser system for upcoming optical injection experiments in the resonant regime.
\end{abstract}

\section{INTRODUCTION}

The concept of optical injection of electrons in laser-produced plasma waves is a promising scheme to commence the second generation of Laser Wakefield Accelerators (LWFA) as it alleviates the shortcomings of the self-injected LWFA traditionally dubbed the Self-Modulated LWFA (SMLWFA). In the latter scheme, a single laser pulse of power above the critical power for Relativistic Self-Focusing (RSF), $P_{c}[\mathrm{GW}]=2 m_{e}^{2} c^{5} n_{c} / \pi e^{2} n_{e} \approx 17 n_{d} n_{e}$, and pulse duration much longer than the plasma wave period ( $\tau_{\text {laser }} \gg 2 \pi / \omega_{p}$ ), impinges upon an underdense target. The relatively long laser pulse gives rise to the onset of Raman Forward (RF), and Raman Backward instabilities. The former excites a fast forward plasma wave, while the latter excites a backward slow wave that is considered the primary [1] mechanism for

CP647, Advanced Accelerator Concepts: Tenth Workshop, edited by C. E. Clayton and P. Muggli (C) 2002 American Institute of Physics 0-7354-0102-0/02/\$19.00 
injecting electrons into the forward wave. As such, the acceleration mechanism is initiated from noise and grows uncontrollably, which results generally in a picosecond-long accelerated electron bunch with an exponentially broad energy distribution, which in turn leads to further temporal broadening. While these parameters are useful for some scientific applications [2], they fall short of fulfilling other more demanding applications [3]. To recap, a new generation of LWFA must surmount (1) the long ps bunch duration (2) the broad energy distribution. A novel concept was proposed by Umstadter et al. [4] acronymed LILAC for Laser Injected Laser ACcelerator, has promising prognosis to overcome the deficiencies with the SMLWFA. The LILAC scheme encompasses a powerful short laser pulse, comparable in duration to the plasma wave period $\left(\tau_{\text {laser }} \approx 2 \pi / \omega_{p}\right)$, loosely focused onto an underdense plasma target. This pulse, called pump pulse, resonantly excites a plasma wave via its ponderomotive action. The excited wakefield is described as 1-D due to the focusing geometry, and is more favorable for particle acceleration for many reasons. In this parameter regime, some plasma instabilities are strongly suppressed, like RSF and RF instabilities. While the former is rather beneficial for prolonging the acceleration length, the suppression of latter is desirable. In addition, the wakefield is not expected to (and was demonstrated not to) self-trap background particles if the wave-breaking limit is not reached [5][6]. This inhibits the dark current and enhances the signal-to-noise ratio of an injected electron bunch into the plasma wave. In order to cause the oscillating background electrons forming the plasma wave to become trapped then accelerated, the phase relation between the position and momentum of these electrons must be optimized for that. In other words, the orbits of the un-trapped electrons in the phase space must be redirected to cross the separatrix and become trapped then co-move with the plasma wave bucket. Numerical simulation of LILAC suggests that this dephasing can be introduced by a second similar pulse but otherwise tightly focused in such a way that its ponderomotive impulse will "kick" the electrons into the right acceleration phase of the plasma wave, this pulse is called injection pulse. Fig. 1 depicts a sketch of the LILAC geometry, and simulation data. The LILAC scheme also suggests that dephasing can be achieved by an ionization "kick" induced by the injection pulse, wherein the presumably more intense injection pulse accesses a deeper ionization state that the pump pulse wont.
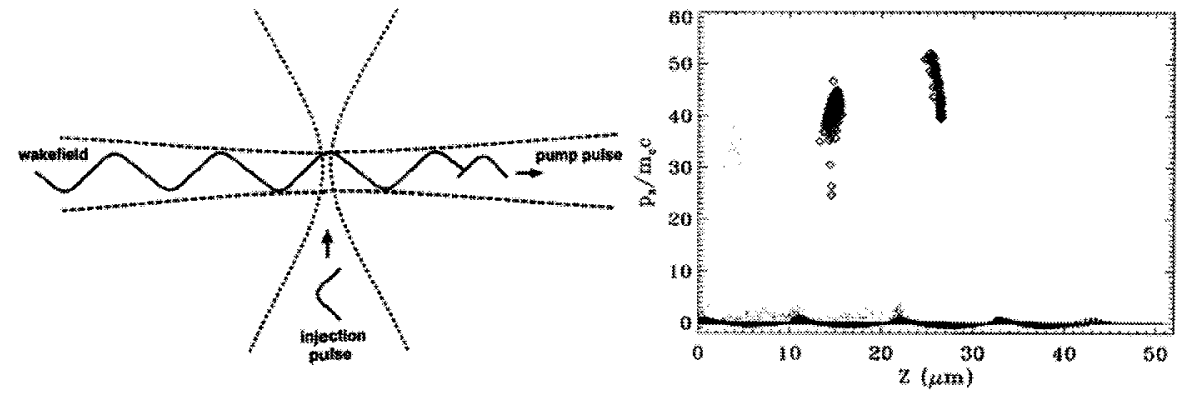

FIGURE 1. (Left) LILAC scheme. (Right) PIC simulation of injection action when injection pulse equals two plasma periods $\left(\tau_{i n j}=2 \tau_{p}\right.$ ), which fills two acceleration buckets with a few-percent energy-spread electron beam. 
The original LILAC scheme has inspired a number of variant schemes using multiple laser pulses [7], or even using one laser pulse [8] but utilizing plasma irregularities to cause dephasing of the electron motion. None of these schemes has been experimentally realized.

\section{PROOF-OF-PRINCIPLE EXPERIMENT}

The implementation of the LILAC scheme requires a short-pulse high-power laser, which is currently under construction at the FOCUS center at the University of Michigan, and to be commissioned soon, see the description below. However, a proof-of-principle experiment can be performed in the meantime using an already existing laser system, a table-top Tera-Watt Nd:Glass system, with $\lambda_{\text {laser }}=1$ in, $\tau_{\text {laser }}=400 \mathrm{fs}$ and maximum output power of $12 \mathrm{TW}$. We estimate the on-target normalized intensity to be $a_{p u m p}=0.5$ and $a_{\text {injection }}=1$. Fig. 2 shows schematic of the experiment, where the incoming main beam from the laser system is horizontally polarized, and split by a 1:4 beam-splitter to pump beam (transmitted at $\% 20$ ), and injection beam (reflected at \%80), respectively. The polarization of the two arms is then rotated by separate half-wave plates to vertical polarization suitable for forming an interference pattern in the plasma target.

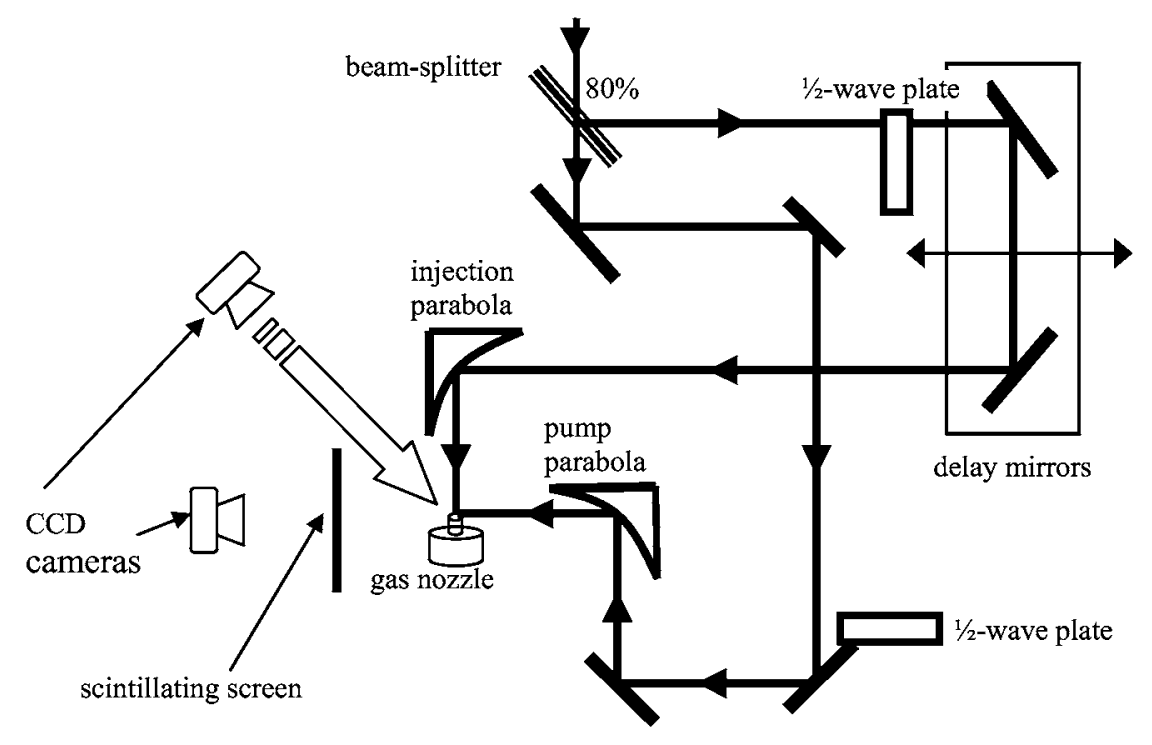

FIGURE 2. Schematic of the long-pulse LILAC experiment.

The two beams intersect in a 1-mm diameter He supersonic gas jet at an underdense plasma density of $n e / n c=0.04$, where $n e$ and $n c$ are the ambient and critical densities, respectively. At this ambient density, the laser pulse straddles about 15 plasma 
periods, which sets it very far from the resonant regime discussed above. The intersection of the two laser beams creates a ponderomotive grid caused by the interference fringes and is interspaced by $\lambda_{\text {laser }} / \sqrt{2} \approx 0.7 \mu \mathrm{m}$. These beamlets remain stationary in space for the entire laser pulse duration till the intersecting pulses bifurcate away from each other. During this time, the electrons in the intersection region get trapped in the ponderomotive potential wells, which act like a periodic deriver that heats the electrons and gives rise to a Fermi-wall-like problem, where at a certain input intensity threshold, the electron motion becomes chaotic, and Stochastic heating takes place. The increase in randomized motion leads to a huge temperature increase, one of the highest plasma temperatures that can be prepared in a laboratory. Heating the trapped electrons broadens their orbits in the phase space until they eventually overlap with the plasma wave separatrix and get picked up by it shortly after the laser pulses have passed each other. The heated electrons are "flushed" in the plasma wave behind the laser pulse since the plasma wave is longer than the laser pulse itself [9]. This enhancement of trapping in the plasma wave driven by the pump pulse induced by virtue of an ancillary laser pulse is another case of optical injection. As a matter of fact, this injection mechanism is yet a new one [10] that was not reported before as such, even though Stochastic heating per se has been observed and simulated ubiquitously in the literature [11]. We have simulated these experimental conditions using a 2-D PIC code. This code simulates two beams of normalized intensities $a_{\text {pump }}=0.25$ and $a_{\text {injection }}=1$. The pulse duration was 40 laser cycles with transverse pulse dimensions of a few 10's of $\lambda_{\text {laser. }}$ The simulation box is $100 \times 60$ $\lambda^{2}$ laser, and the beams propagate through a homogonous plasma background density of $0.04 n_{c r}$. Absorption boundaries in both $\mathrm{x}$ - and $\mathrm{y}$-directions are adopted for the fields. Particles reaching $x-y$ boundaries are reflected with initial thermal velocities, 10 cells per $\lambda_{\text {laser }}$ and 9 particles per cell were used throughout the simulation, and ions are kept fixed due to their inertia. The polarizations of the two beams were chosen to be pointing in the z-direction perpendicular to the propagation vectors of the two beams in one run, and parallel to the plane of propagation in another run. This was done to study the effect of the interference of the two beams, since the interference should vanish with the interference term $\left(\boldsymbol{E}_{1} \bullet \boldsymbol{E}_{2}\right)$. Fig. 3 shows the results of the simulation at $t=40 \tau_{\text {laser }}$ when the beams are just fully overlapped over their nominal pulse durations. The modulation of the density, $\Delta n_{\mathrm{e}}$, is shown to be more than 10 times the background density, this is more than an order of magnitude of the highest measured plasma wave density modulation [12][13] that corresponds to the relativistic cold plasma wavebreaking limit. To our knowledge, this is the highest relative density modulation in under-dense plasma ever reported to date. We can use the results of the 2-D PIC simulation to establish a figure of merit for the average electric field associated with this density modulation that was qualitatively measured by Thomson Scattering (TS) in our experiment. This can be done using Poisson's law with a pillbox surrounding an electron bunch sheet in this volume grating, which immediately gives $E=e \Delta n_{e} L / \varepsilon_{0} \approx 1 \mathrm{GV} / \mathrm{mm}$, with $L$ being the scale length $(\approx 0.7 \mu \mathrm{m})$. This field is a stationary DC electric field on average over the nominal pulse duration, and it vanishes around some zero-potential point between any two consecutive charge 
sheets, giving rise to a record sharp electric field gradient, $d E / d x$, on the order of 1 $\mathrm{TV} / \mathrm{mm}^{2}$. Thus, this experiment reports an estimated highest average electric field and field gradient ever prepared in a laboratory experiment with under-dense plasmas. Notwithstanding these extreme conditions, their effect outside the overlap region rapidly peters out, primarily due to the localized nature of the density modulation structure produced, and the quasi-neutrality of the plasma owing to the uniform ion background distribution in this short pulse regime.

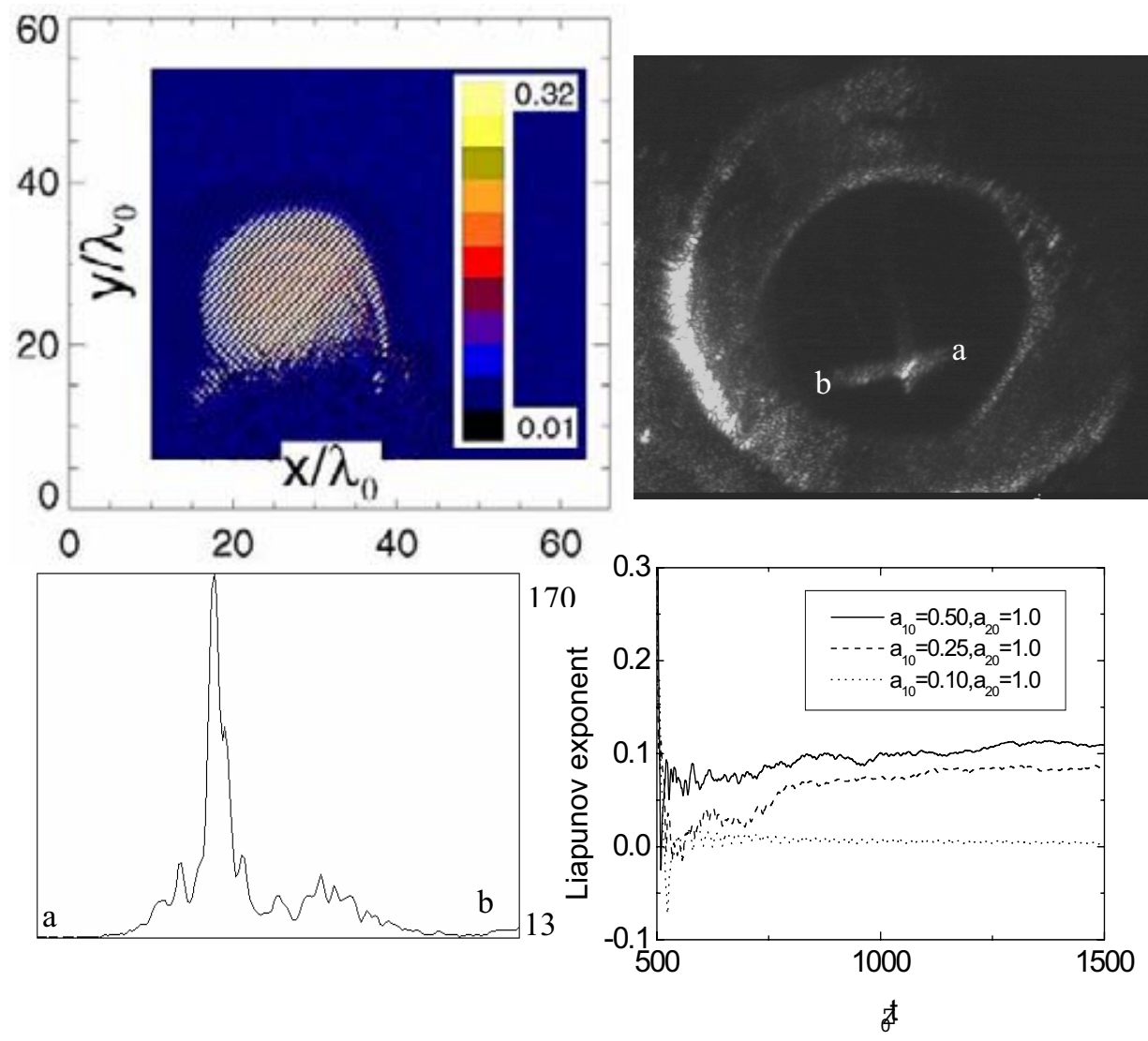

FIGURE 3. (Top left) PIC code simulation of the plasma density modulation as the two laser beams cross. Pump beam along the positive $\mathrm{x}$-direction and injection beam along the positive $\mathrm{y}$-direction. The density modulation is more that $10 \mathrm{X}$. (Top right) Experimental observation of Thomson scattering imaged with a CCD camera from the top of the gas nozzle. (Bottom left) Lineout of the contrast from point (a) to point (b) shows more than $10 \mathrm{X}$ enhancement of Thomson scattering at the overlap region. (Bottom right) Liaponuv exponent for different normalized intensities for pump and probe. Positive value indicates stochastic motion.

The simulation also shows, Fig. 4. (a), that the temperature of the electron beam accelerated by the plasma wave driven by the pump beam is enhanced by more than an order of magnitude when it is overlapped with the injection beam. This sets a 
unique method of injection and coherent control where, not only the electron energy is increased, but also the electron beam temperature is. Our simulation results suggest that the ponderomotive fringes constitute an optical trap 10 times higher than the optical trap reported to date [14]. Furthermore, the simulation reveals the onset of chaos and stochastic heating of the electrons trapped in the ponderomotive wells. This type of motion has been numerically observed before [15] in the single particle regime. We have performed calculations of the Liapunov Parameter (LP) to investigate the occurrence of stochasticity. Fig. 3. shows that indeed the LP has positive values for our experimental parameters that persist even after the pulses have overlapped. No wavebreaking has been observed in the simulation during the time where the two pulses were overlapped. Fig. 4(b) shows that the electrons are chaotically heated during the overlapping of two pulses, while the amplitude of the plasma wave is below wavebreaking despite the existing huge stationary density modulation. The two density modulations, the propagating (plasma) modulation, and the stationary one, superimpose on each other. The experimental results agree with the simulation. Fig. 3. (top right) shows the image captured by a CCD camera with a band-pass filter of $(1064 \pm 5) \mathrm{nm}$ placed in front of it.

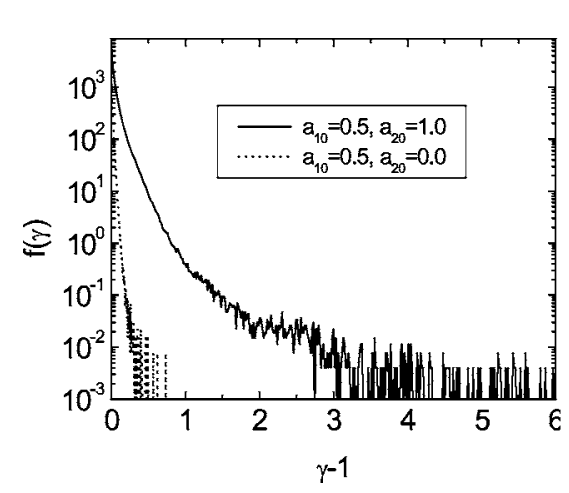

(a)

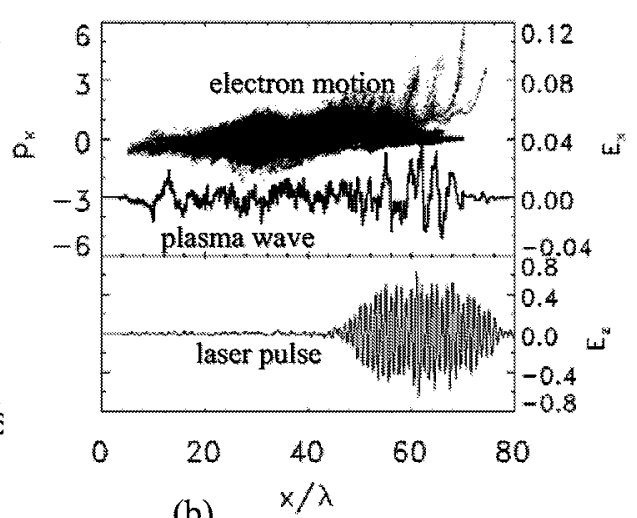

(b)

FIGURE 4. (a) Electron beam energy distribution function of the electrons accelerated by the pumpbeam-driven plasma wave. The dotted curve is without the injection beam and the solid curve is with the injection beam on. Enhancement of the temperature (1/slope) is observed. Compare this to the experimental observation in Fig. 5. (right) (b) Simulation of the laser pulse, the plasma wave amplitude is normalized to $\left(m_{e} \omega c / e\right)$, and electron momenta are normalized to $\left(m_{e} c\right)$.

The Thomson-scattered light exhibits significant enhancement along the bisector direction. This enhancement is attributed to single-particle and collective effects. In the single particle picture, the radiation cross-section, $\partial \sigma_{\text {rad }} \partial \Omega$, is enhanced in the crossed-field region. The radiation cross-section can e written as

$$
\frac{\partial \sigma_{r a d}}{\partial \Omega} \propto|(\hat{\mathbf{n}} \times \dot{\mathbf{v}}) \times \hat{\mathbf{n}}|^{2} \propto \sin ^{2} \Theta_{\hat{\mathbf{n}}, \dot{\mathbf{v}}}
$$



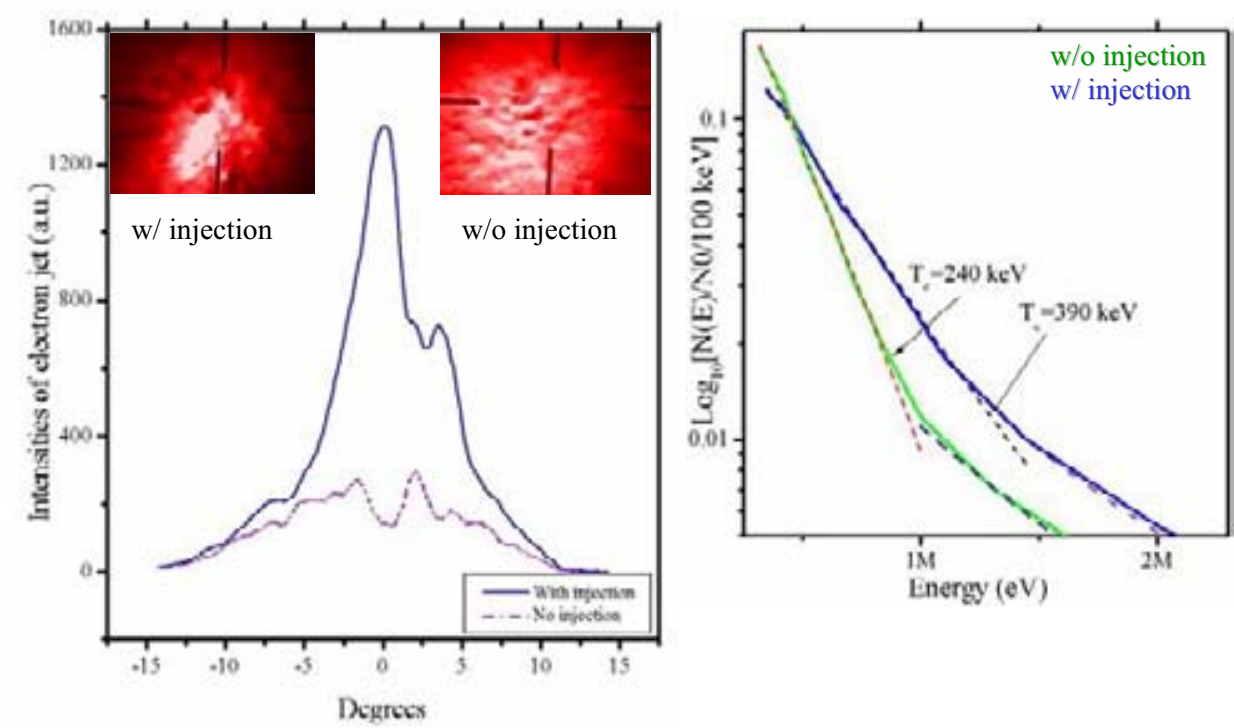

FIGURE 5. (Left) Pump electron beam transverse distribution imaged on a scintillating screen in the pump forward direction with lineout representative of the current and emittance enhancement. (Right) Pump electron beam energy distribution, temperature enhancement is shown to be about $50 \%$.

Here, $\hat{\mathbf{n}}$ is the observation direction, $\dot{\mathbf{v}}$ is the acceleration of the particle, and $\Theta_{\hat{\mathbf{n}}, \dot{v}}$ is the angle between them. In the single beam case, and using the plane wave approximation for a plane wave propagating in the y-direction, the equation of motion (EOM) can be approximately written to the first order as

$$
\frac{d \mathbf{v}}{d t} \approx \frac{e}{m_{0}}(\mathbf{E}+\mathbf{v} \times \mathbf{B})=\hat{\mathbf{z}} \frac{e E_{0}}{2 m_{0}} \cos (k y-\omega t)+\hat{\mathbf{y}}(\text { drift term })
$$

In our experiment, the observation direction was along the z-direction. Thus, the cross product in Eq. (1) vanishes. In the overlap region, however, the EOM can be written as

$$
\begin{gathered}
\mathbf{E}=\hat{\mathbf{z}} E_{z}=\hat{\mathbf{z}} E_{0}(\cos (k x-\omega t)+\cos (k y-\omega t)) \\
\frac{d \mathbf{v}}{d t} \approx \frac{e}{\gamma m_{0}}(\mathbf{E}+\mathbf{v} \times \mathbf{B})=\hat{\mathbf{z}} \frac{e E_{z}}{m_{0}}+\left(\frac{e E_{0}}{\not m_{0} \omega c}\right)^{2} \omega c(\hat{\mathbf{E}} \times \hat{\mathbf{B}}) \\
\hat{\mathbf{E}} \times \hat{\mathbf{B}}=(\hat{\mathbf{x}} \cos (k x-\omega t)+\hat{\mathbf{y}} \cos (k y-\omega t))
\end{gathered}
$$

Evidently, the second term in Eq. (3) for $d \mathbf{v} / d t$ enhances the radiation cross section due to the component in the $x-y$ plane. The collective effects emerge from the 
balancing of the ponderomotive force in the interference fringes, and both the space charge and electron temperature effects. The mathematical treatment of the collective effects is beyond the scope of this manuscript due to its intractability. Finally, the enhancement of the pump electron beam current and emittance when the injection pulse is turned on has been observed and shown in Fig. 5.

\section{LASER SYSTEM DEVELOPMENT OF SHORT PULSE 100 TW FOR RESONANT LILAC EXPERIMENT}

We are developing a $100 \mathrm{TW}$-class laser system capable of producing pulses as short as $30 \mathrm{fs}$ suitable for optical injection experiments in the resonant regime $\left(\tau_{\text {laser }} \approx 2 \pi\right.$ $\left(\omega_{p}\right)$. The development of the laser system went through two different phases. In the first phase, the laser system is described in [16][17]. The laser system was successfully commissioned and a series of experiments was conducted and reported [5][18]. A few significant steps towards materializing the resonant LILAC scheme were established, which helped narrow down the parameter space in which the proposed experiment should run. Fig. 6. displays the resonant wakefield produced by a $6 \mathrm{TW}, 28 \mathrm{fs}$ pulse, measured by collective collinear Thomson scattering of a frequency-doubled probe. In addition, a pump power range was identified for the pulse that produces the resonant wakefield so that filamentation instability can be avoided. Furthermore, no dark current was detected in the resonant regime, a result that was later reported by [6], too. No further experimentation was conducted to inject a second laser beam into the produced resonant wakefield. The second phase of the laser development encompasses a radical redesign of the laser system, the new laser system is called HERCULES Laser System (HLS). The HLS is a high-contrast, 100TW-class laser system based on Chirped Pulse Amplification (CPA) technique [19].
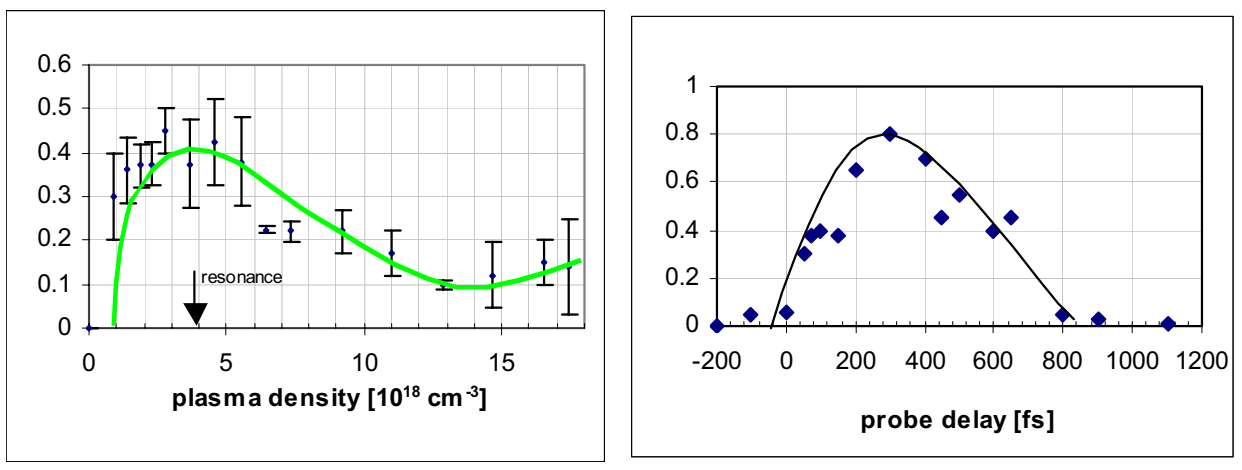

FIGURE 6. (Left) Resonant wakefield measured by $2 \omega$ Thomson probe. (Right) Wakefield duration. The vertical axis in both plots is the amplitude of the Thomson probe in arbitrary units.

As shown in Fig. 7., the system consists of an oscillator that puts out a 12 fs pulse, which is then cleaned (using a saturable absorber) and pre-amplified to enhance the contrast. The pulse after that is stretched via a $100 \mathrm{~nm}$ throughput, grating-mirrorstripe based stretcher. This arrangement jibes with the compressor gratings 
arrangement to allow for a quintic-phase-limited compression where at the working point in the configuration space, all dispersion orders below the fifth are practically zeroed. After the pulse is stretched to $\sim$ ns with about $50 \mathrm{~nm}$ bandwidth, it is then injected into a novel 7.5-meter-cavity ring regenerative amplifier (RRA) [20]. The RRAs have distinctive advantages over the linear ones in terms of energy throughput, isolation from the oscillator, and pulse contrast compared to the amplified spontaneous emission, but yet has a smaller footprint. An output pulse of the RRA, which can reach up to $90 \mathrm{~mJ}$, is then injected into a 4-pass amplifier whose 3-cmdiameter Ti:Sapphire crystal is cryogenically cooled to $120^{\circ} \mathrm{K}$ which practically eliminates thermal distortions. Fig. 8. depicts the transverse beam profiles at $10 \mathrm{TW}$ after the 4-pass amplifier, and Fig. 9 show the pulse duration measurement using a single-shot auto-correlator. The final amplification stage utilizes a 10 -cm-diameter Ti:Sapphire crystal pumped by a $15 \mathrm{~J} \mathrm{Nd:YLF} \mathrm{glass} \mathrm{laser} \mathrm{system.} \mathrm{This} \mathrm{amplifies} \mathrm{the}$ final beam to a few-100-TW level after which it gets split and sent to two identical but separate compressors that allow for different pulse compression suitable for pump-probe, or pump-injection experiments. The final compressed output pulse is transform-limited in duration to about $30 \mathrm{fs}$.

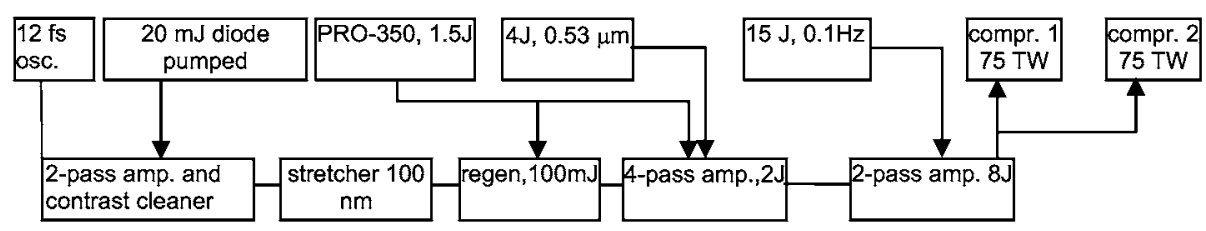

FIGURE 7. Schematic of the HERCULES laser system, the sub-systems on top are the pump lasers, and the lower line of systems are amplification systems. The output pulse is split to two compressors.

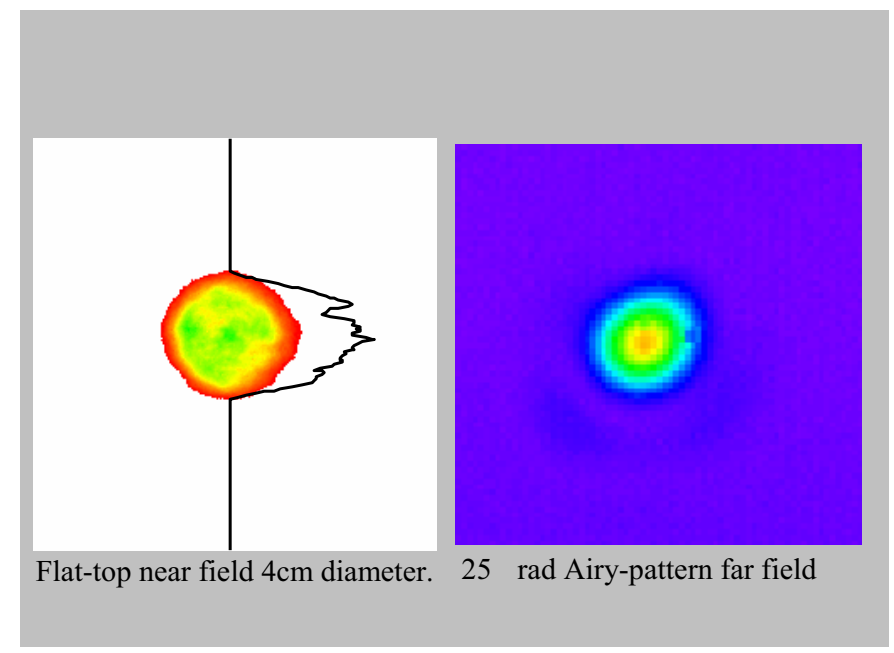

FIGURE 8. Near- and far-field transverse beam measurement imaged on a CCD from a pick-off mirror at $10 \mathrm{TW}$. 


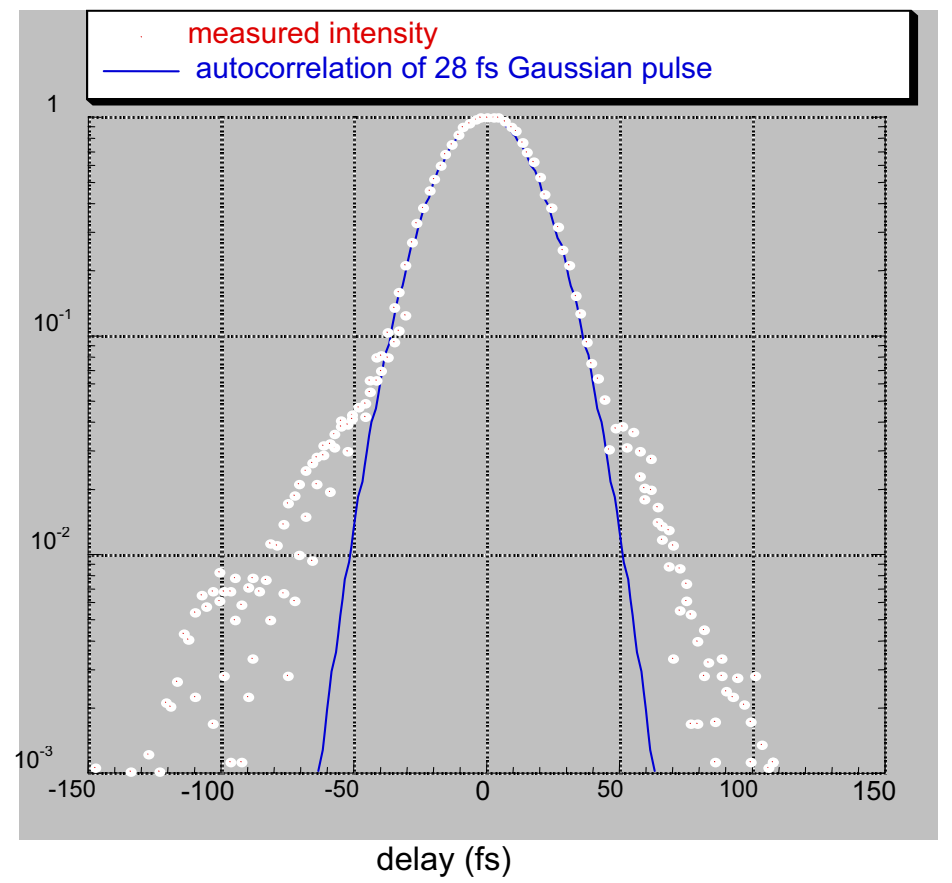

FIGURE 9. (dots) Single-shot autocorrelation pattern of the output beam after the 4-pass amplifier at $10 \mathrm{TW}$ compared to a Gaussian beam (solid line), the pulse duration is measured to be $28 \mathrm{fs} \mathrm{FWHM.}$

\section{ACKNOWLEDGEMENT}

Many thanks to G. Mourou, P. Rousseau, V. Chvykov, and G. Kalinchenko for their contribution to the laser system. This work is supported by U.S. DOE, award DEFG02-98ER41071, with lasers supported by NSF. 


\section{REFERENCES}

1. P. Bertrand et al., Physical Review E. 49, 5656 (1994).

2. N. Saleh et al., Rev. Sci. Instr. 71, 2305 (2000).

3. A. D'Angelo et al., NIM. A 445, 1-6 (2000).

4. D. Umstadter et al., Phys. Rev. Lett. 76, 2073 (1996).

5. X. Wang et al., Phys. Rev. Lett. 84, 5324 (2000).

6. V. Malka et al., Physics of Plasmas 8, 2605 (2001).

7. E. Esarey et al., Phys. Rev. Lett. 79, 2682 (1997), B. Rau et al., Phys. Rev. Lett 78, 3310 (1997), R. G. Hemker et al., Phys. Rev. E. 57, 5920 (1998), C.I. Moore et al., Phys. Rev. Lett. 82, 1688 (1999).

8. S. Bulanov, Plasma Phys. Rep. 25, 468 (1999), H. Suk et al., Phys. Rev. Lett. 86, 1011 (2001).

9. S. P. Le Blanc et al., Phys. Rev. Lett. 77, 5381 (1996).

10. P. Zhang et al., to be published in Phys. Rev. Lett.

11. Z.-M. Sheng et al., Phys. Rev. Lett. 88, 055004 (2002).

12. D. Umstadter et al., Science 273, 472 (1996).

13. A. Ting et al., Phys. Rev. Lett. 88, 5377 (1996).

14. J. Chaloupka et al., Phys. Rev. Lett. 83, 4538 (1999).

15. D. Bauer et al., Phys. Rev. Lett. 75, 4622 (1995).

16. S. Backus et al., Review of Scientific Instruments, 69, 1207 (1998).

17. H. Wang et al., JOSA B, 16, 1790 (1999).

18. N. Saleh et al., "Status of LILAC Experiment" in AIP Conf. Proc. 569, 127 (2001).

19. V. Yanovsky et al., Technical Digest of CLEO 2000, 288 (2000).

20. V. Yanovsky et al., Applied Physics B. 74181 (2001). 